\title{
Investigation of a Mitigation Strategy for Thermal Effects of $X$-ray Sources in Computed Tomography
}

\author{
Felix Binder ${ }^{1}$, Benjamin Baumgärtner ${ }^{1}$, Tino Hausotte ${ }^{1}$ \\ ${ }^{1}$ Institute of Manufacturing Metrology (FMT), Friedrich-Alexander-University Erlangen-Nuremberg \\ (FAU), \\ felix.binder@fmt.fau.de
}

\begin{abstract}
Summary:
Computed tomography (CT) is a fast and non-destructive measurement method with an increasing industrial application area that demands powerful X-ray sources. Depending on factors, like the measurement settings, the environmental conditions and the effectiveness of the installed cooling system, the dissipated heat of the X-ray source can influence the measurement. In this contribution a mitigation strategy will be described, which approximates the thermal behaviour of an X-ray source in order to derive a task specific warm-up time that reduces the temperature difference during a CT measurement.
\end{abstract}

Keywords: computed tomography, temperature effects, mitigation strategy, thermal characterization, X-ray source

\section{Introduction}

Dimensional measurements in $\mathrm{X}$-ray computed tomography (CT) are often carried out under stable environmental conditions to disregard temperature changes during a measurement. With increasing power consumption of industrial X-ray sources, it is reasonable to investigate the thermal behaviour of such a component.

However, a complete thermal characterization requires detailed knowledge of all active components, the cooling system, the materials, the geometrical setup and the measurement parameters. The presented approach focuses on an alternative way to approximate the thermal behaviour of an X-ray source in order to calculate a task specific warm-up time $\Delta t_{\mathrm{W}}$, which reduces the total temperature difference $\Delta \vartheta$ during the critical part of a CT measurement.

\section{Theory}

The majority of the used power to produce Xrays is dissipated as heat into the CT system, which influences the stability of the projected images [1], presumed that the cooling system cannot totally compensate the heat. Furthermore, temperature variations of the X-ray source can cause geometrical displacements [2], which result in translation, rotation and dilation errors of the reconstructed volume [3].

Therefore, it is assumed that a reduction of the total temperature difference $\Delta \vartheta$, caused by the $X$-ray source, will also reduce geometrical displacements during the measurement. Based on that assumption, an additional warm-up time $\Delta t_{\mathrm{W}}$ is considered to improve a cold-start measurement procedure as described in Fig. 1.

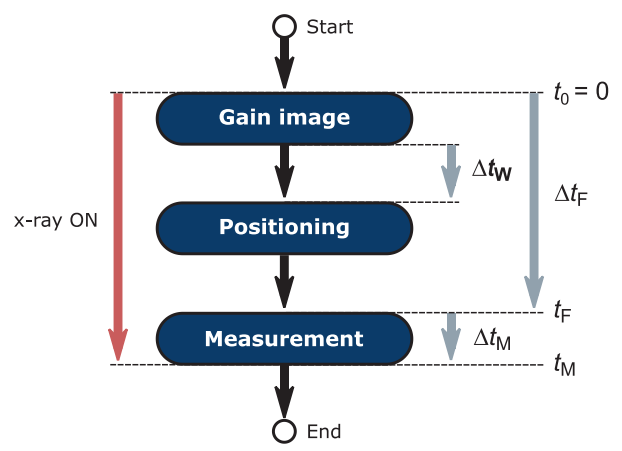

Fig. 1. Cold-start measurement procedure including an additional time $\Delta t_{\mathrm{W}}$ to warm-up the system.

The gain image acquisition and object positioning phase can be summarized as a pre-heat phase with the duration $\Delta t_{\mathrm{F}}$. During this phase, the $\mathrm{X}$-ray tube will heat-up until $t_{\mathrm{F}}$ is reached and the actual thermal sensitive measurement starts for the duration of $\Delta t_{\mathrm{M}}$ and ends at $t_{\mathrm{M}}$. Since $\Delta t_{\mathrm{W}}$ will increase the overall measurement time, but reduces the temperature difference $\Delta \vartheta$, different strategies can be derived to find a task specific optimum.

\section{Experiment}

A Pt100 contact sensor, which has been corrected with a calibrated reference thermometer, has been installed on the housing of the X-ray tube, close to the reflection target (tungsten), which is known to be the main heat source of the system. The sensor temperature was logged 
every $5 \mathrm{~s}$. The X-ray tube has a peak power consumption of $500 \mathrm{~W}$ and is actively water-cooled. Additionally, the tube is indirectly air-cooled by two radiators, that provide ventilation of cooled air from the surrounding measurement room with a set temperature of $(20.0 \pm 0.2){ }^{\circ} \mathrm{C}$ and a relative humidity of $(45 \pm 10) \% \mathrm{RH}$. A series of measurements, across the operating power range of the X-ray tube have been carried out according to the parameters in Fig. 2.

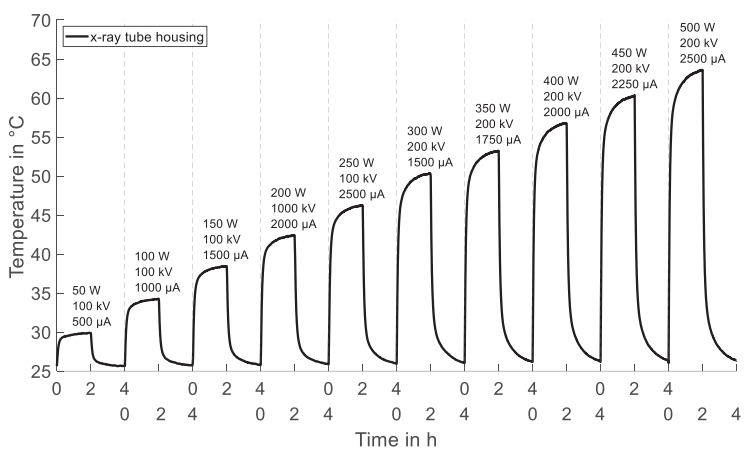

Fig. 2. X-ray tube housing temperature of a measurement series with increasing power consumption.

For the characterization of each measurement, a suitable fit function (1) has been used to approximate the constant $t_{\mathrm{c}}$.

$\vartheta(t) \approx \vartheta_{0}+\left(\vartheta_{\mathrm{eq}}-\vartheta_{0}\right) \cdot e^{-\frac{t_{\mathrm{c}}}{t}}$

$\vartheta_{0}$ denotes the common base temperature of the system and $\vartheta_{\text {eq }}$ the equilibrium temperature for each measurement. With the determined constants $t_{c}$ the total temperature difference $\Delta \vartheta$ can be calculated with (2) according to the measurement procedure defined in Fig. 1.

$\Delta \vartheta=\left[\vartheta\left(t_{\mathrm{M}}\right)-\vartheta\left(t_{\mathrm{F}}\right)\right]-\left[\vartheta\left(t_{M}^{\prime}\right)-\vartheta\left(t_{F}^{\prime}\right)\right]$

$t_{\mathrm{F}}^{\prime}$ and $t_{\mathrm{M}}^{\prime}$ describe the times on which the preheat and the measurement phase ends, considering an additional warm-up time $\Delta t_{\mathrm{W}}>0$.

\section{Results}

Approximation (1) and equation (2) are used to estimate the total temperature difference $\Delta \vartheta$ for $\Delta t_{\mathrm{W}} \in[0,500] \mathrm{s}$ with $\Delta t_{\mathrm{F}}=230 \mathrm{~s}$ and $\Delta t_{\mathrm{M}}=$ 6300 s. $\Delta t_{\mathrm{F}}$ and $\Delta t_{\mathrm{M}}$ are task specific values, that depend on the number of gain images, the number of projection images, the integration time of the detector and a CT specific delay (e. g. rotating the axis or image processing). In this case $\Delta t_{\mathrm{F}}$ and $\Delta t_{\mathrm{M}}$ were assumed for a precise measurement with 90 gain images, 1500 projection images and a detector integration time of $2000 \mathrm{~ms}$.

The resulting temperature differences $\Delta \vartheta$ for each power setting are summarized in Fig. 3 and show that without an additional warm-up time a significant temperature difference during the measurement still can be expected. Furthermore, the gradient and therefore the optimization potential increases with the power consumption.

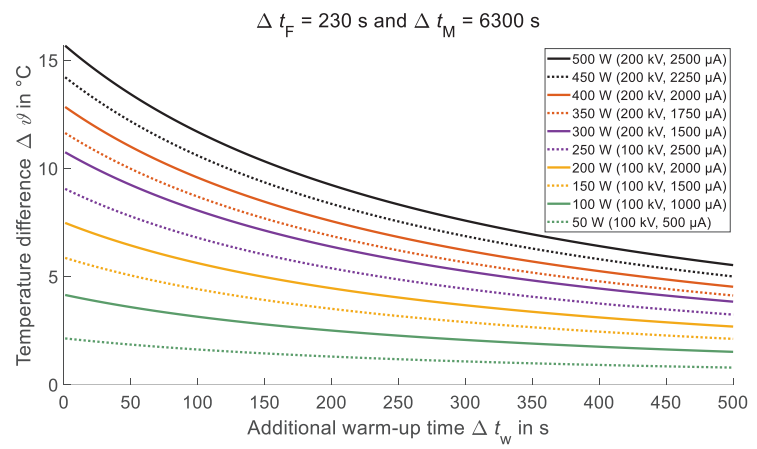

Fig. 3. Temperature differences for each power setting with an additional warm-up time $\Delta t_{\mathrm{W}}$.

\section{Conclusion}

An investigation has been carried out, which is based on a series of temperature measurements across the operating range of the tube. The logged temperatures can be used to approximate the thermal behaviour of the X-ray source for the given parameter settings.

Even though a pre-heat phase has been considered, the results show that with increasing power consumptions an increasing temperature difference $\Delta \vartheta$ can be expected, which may influence the measurement if uncompensated. Integrating a task specific additional warm-up time $\Delta t_{\mathrm{W}}$ as a countermeasure, for example by increasing the number of gain images, yields the potential to reduce thermal effects caused by the X-ray source for a small increase of total measurement time.

\section{Acknowledgement}

This work is part of the European Metrology Programme for Innovation and Research (EMPIR) project 17IND08 AdvanCT. The EMPIR initiative is co-funded by the European Union's Horizon 2020 research and innovation programme and the EMPIR participating states.

\section{References}

[1] W. Sun, S. Brown, N. Flay, M. McCarthy, J. McBride, A reference sample for investigating the stability of the imaging system of $x$-ray computed tomography, Measurement Science and Technology 27, doi: 10.1088/0957-0233/27/8/085004

[2] M. Katic, N. Ferdelji, D. Sestan, Investigation of Temperature-Induced Errors in XCT Metrology, International Journal of Automation Technology 14, 484-490 (2020); doi:10.20965/ijat.2020.p0484

[3] N. Limodin, J. Rethore, J. Adrien, J. Y. Buffiere, F. Hild, S. Roux, Analysis and Artifact Correction for Volume Correlation Measurements Using Tomographic Images from a Laboratory X-ray Source, Experimental Mechanics 51, 959-970 (2011); doi: https://doi.org/10.1007/s11340-0109397-4 